\title{
Advanced Window Grids for Work at the Intersection of Electron and Optical Imaging
}

\author{
C.C. Striemer* and T.R. Gaborski*
}

* SiMPore, Inc., 150 Lucius Gordon Drive, Suite 100, West Henrietta, NY 14586

It is becoming increasingly common for researchers to use multiple imaging techniques when characterizing cells, nanoparticles, large molecules, and small molecule dyes, particularly in systems where these materials interact. For example, fluorescence microscopy is very effective in characterizing the general location of biochemical events within a cell, but has limited resolution for discerning fine structures. Electron microscopy has superior resolution, but often requires substrates that are foreign or incompatible with cell culture, leading to experimental compromises and complications. To meet this need, SiMPore has created a range of substrates and a product platform to facilitate the use of both optical and TEM/SEM analysis in the biological laboratory.

Responding to customer inquiries, the $\mathrm{TEMVu}^{\mathrm{TM}}$ optical slide platform has been developed to meet the needs of researchers who need to implement electron imaging in biological research and is pictured in figure 1A. The autoclaveable polycarbonate frame is the size of a standard glass microscope slide, but has a small fluidic chamber with a $3 \mathrm{~mm}$ TEM grid. The floor of this chamber is a glass coverslip for optimal optical imaging. The chamber can either be completely flooded with media or small volumes of different solutions can be placed on each side of the grid for greater flexibility. When sample preparation is complete, the grid is released from its mount and placed in the TEM or SEM for subsequent imaging. TEMVu ${ }^{\mathrm{TM}}$ slides are available with a range of mounted TEM window substrates including UltraSM ${ }^{\circledR}$ porous nanocrystalline silicon [1], $\mathrm{SiO}_{2}$, and $\mathrm{SiN}$ membrane materials with various thicknesses and geometries to meet specific research needs. All ultrathin window grids are extremely transparent optically. A wide range of custom solutions have also been developed for customers.

Figure 1B shows an SEM image of a human neutrophil placed on an IL-8 coated UltraSM ${ }^{\circledR}$ pure silicon TEM window grid (15 nm thick) and allowed to activate. Cells were fixed with glutaraldehyde and then dried with increasing concentrations of ethanol. Pure silicon TEM windows are excellent substrates for electron microscopy, as the higher conductivity of silicon films provides for better charge dissipation than oxide or nitride films, eliminating the need for additional gold sputtering. This image required no sample prep beyond drying.

In addition to cell culture, the UltraSM ${ }^{\circledR}$ porous nanocrystalline silicon membrane material has a range of applications in high resolution TEM where nanoscale objects need to be suspended across open holes in a thin support film to avoid interference with the underlying substrate. Suspending nanoscale objects over nanoscale holes offers the highest probability of successful placement and provides the best support to the suspended material. SiMPore has offered this type of grid for over 2 years, and has recently introduced an improved version with $3 \mathrm{X}$ greater porosity and greater pore size for increased field of view for the suspended objects, as shown in Figure 2.

An overview of these products, their advantages and enabling applications will be presented. 


\section{References}

[1] C.C. Striemer, Nature. 445 (2007) 749.

A)

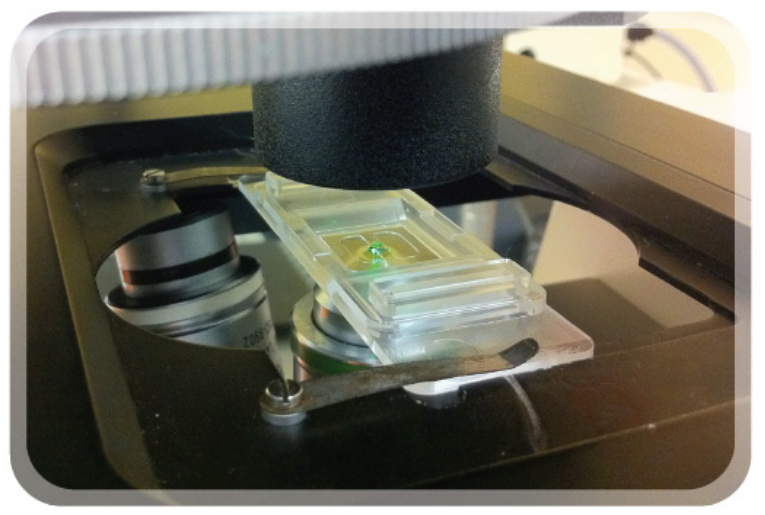

B)

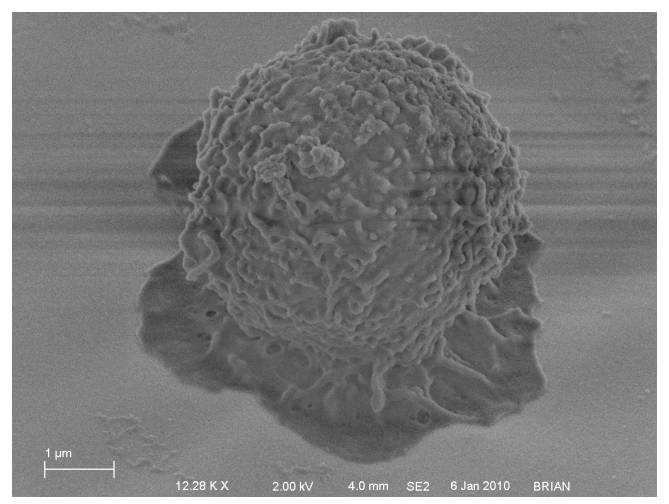

Figure 1. A. TEMVu slide, consisting of a standard 3mm TEM sample mounted in a fluidic slide compatible with cell culture, fluroescence staining, optical characterization, and general TEM sample preperation with fluid media. B. SEM image of a human neutrophil on a $15 \mathrm{~nm}$ thick UltraSM $^{\circledR}$ TEM window. The cell was fixed at the initiation of spreading and prior to imaging (Image Credit: Brian McIntyre and Elena Lomakina, University of Rochester).
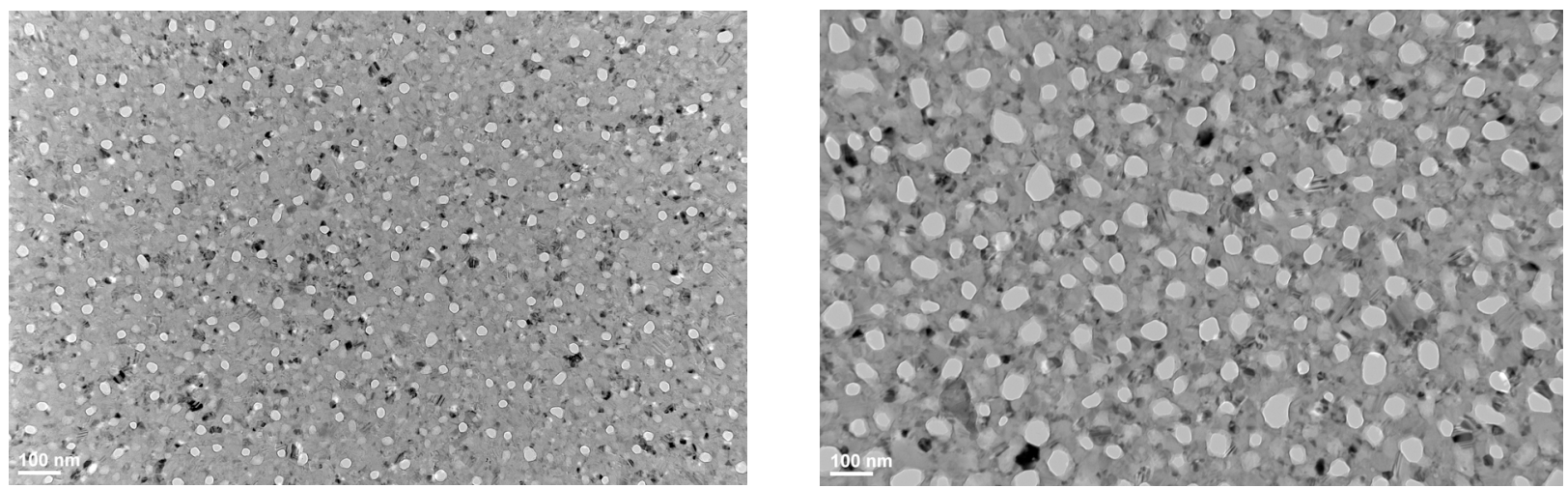

Figure 2. Recent materials improvement in the UltraSM porous nanocrystalline silicon substrate has increased porosity by $>3 \mathrm{X}$ and more than doubled the average pore size. The older material is shown on the left and the improved material is on the right. In these images, the pores are white and the background is composed of randomly oriented silicon nanocrystals with various levels of diffraction contrast. This material is an excellent substrate for optical and electron imaging of cells, providing a porous biocompatible substrate for growth. Nanopores also provide the capability of suspending nanotubes, nanoparticle, graphene sheets, or large biomolecules across open space for high resolution imaging. 